Proc. of the 13th Symposium of Magnetic Measurements and Modeling SMMM'2018, Wieliczka, Poland, October 8-10, 2018

\title{
Magnetic Determination of Ferrite Content in Steels
}

\author{
I. MÉszÁros* And B. BÖGRE \\ Budapest University of Technology and Economics, Department of Materials Science and Engineering, \\ H-1111 Bertalan L. u. 7., Budapest, Hungary
}

\begin{abstract}
In this paper three different magnetic ferrite determination methods were compared. The tested measuring techniques were AC magnetometer, DC magnetometer and a commercial ferritscope device. A model sample set was prepared from 2507 type super-duplex stainless steel by cold rolling and heat treatment. The above-mentioned methods were used to determine the $\delta$-ferrite content of these samples. The results of the different electromagnetic methods were compared with each other. The ferrite phase ratio values determined by AC and DC magnetometers were close to each other in case of all deformation extents and heat treatments. In contrast, the ferritscope device gave significantly lower ferrite contents in case of plastic deformed samples. The stronger the cold rolling reduction was the lower the measured ferrite content was. This phenomenon was explained by the change of the shapes of magnetization curves due to plastic deformation. The limits, disadvantages, and advantages of the applied methods were analyzed.
\end{abstract}

DOI: 10.12693/APhysPolA.136.681

PACS/topics: 01.40.gf

\section{Introduction}

Nowadays, the importance of nondestructive (NDT) magnetic measurements has increased rapidly. The fast and widely useable NDT can detect defects (cracks, voids etc.) or can be used to study material properties without damaging the sample. The magnetic- and electromagnetic measurements are especially useful for determining the microstructural changes of alloys caused by technological- or material deterioration processes due to service. Several NDT methods are used in industrial practice from which those electromagnetic methods are investigated in this paper which are suitable to determine ferrite content of alloys. Alternating current (AC) magnetometer, direct current (DC) magnetometer, and ferritscope were applied to measure the $\delta$-ferrite content of cold rolled and heat treated super-duplex stainless steel (SDSS) samples.

SDSS is a particular category of stainless steels characterized by a double-phase microstructure with about equal proportions of austenite and ferrite phases. The combination of properties, including high strength and excellent corrosion resistance and stress corrosion cracking in chloride ion containing environments make SDSS very attractive for many applications. Unfortunately, SDSS has several disadvantages as well. One of them is associated with a phase transformation process. In duplex stainless steel there is an eutectic decomposition of ferrite phase which means the transformation of the $\delta$ ferrite into sigma phase and secondary austenite due to heat treatment $\left(\delta \rightarrow \sigma+\gamma_{2}\right)[1,2]$. If the well-adjusted ferrite-austenite phase ratio changes due to heat input, the beneficial properties of this alloy disappear. Some

*corresponding author; e-mail: meszaros@eik.bme.hu percentage decrease of the ferrite content can significantly decrease the corrosion resistance and impact energy of SDSS. Therefore, the determination of ferrite content is essential in heat treated or welded duplex stainless steel structures.

The aim of this study was to compare the capabilities of three different electromagnetic methods which are suitable for ferrite content determination.

\section{Tested samples}

For studying the capabilities of the afore mentioned electromagnetic methods the 2507 grade SDSS was chosen as a test material. This SDSS contains about $25 \%$ chromium and $7 \%$ nickel as main alloying elements. From the original sheet material 35 uniform samples were cut with the size of $15 \times 10 \times 100 \mathrm{~mm}^{3}$. Samples were cold rolled at room temperature with six different reduction rates $(0,10.3,22.3,31.3,41.6,50.6,61.9 \%)$. The rolled samples were heat treated at $700^{\circ} \mathrm{C}, 750^{\circ} \mathrm{C}$, $800^{\circ} \mathrm{C}, 850^{\circ} \mathrm{C}$ temperatures for $30 \mathrm{~min}$ and cooled in normal air. At the end of the preparation process all samples were rolled for the same geometry $\left(3.4 \times 10 \times 100 \mathrm{~mm}^{3}\right)$ which was suitable for the applied $\mathrm{AC}$ magnetometer and ferritscope devices. The applied DC magnetometer requires bulk specimens, so the milled samples were cut into more pieces and fixed into a rectangular cuboid $\left(10 \times 10 \times 10.2 \mathrm{~mm}^{3}\right)$.

\section{Applied magnetic measurements}

As it is known, the magnetic saturation polarization is directly proportional to the ferromagnetic phase ratio (ferrite content in our case) of the specimen [3].

The AC magnetometer is suitable to measure the hysteresis and normal magnetization curves of the specimen from which several magnetic descriptors like maximal 
and remnant induction, coercive field, and initial permeability can be derived. In our tests 200 minor hysteresis loops were measured and the normal magnetization curves were determined from the peak points of the minor hysteresis loops. The maximal excitation field of the AC magnetometer was limited by the measuring setup. It was about $12,800 \mathrm{~A} / \mathrm{m}$ which was definitely not enough to saturate the samples. The saturation polarization values were calculated by an extrapolation method based on the multiphase hyperbolic model. The details of the applied AC magnetometer are described in our previous papers $[4,5]$.

The so called Stablein-Steinitz DC magnetometer is a magnetic bridge which has two symmetrical yokes and a small cross-section cross bridge [6]. The details of the applied DC magnetometer are described in our previous paper $[7,8]$.

The maximum excitation field strength was about $270000 \mathrm{~A} / \mathrm{m}$. Therefore, this setup is capable to excite the bulk steel samples into magnetic saturation which makes it one of the most precise ways of the ferrite content measurement. Unfortunately, this setup is not portable and is only for laboratory use because of its extensive size.

Samples were also measured by a commercial FERITSCOPE FMP30 type equipment of the Fischer company [9]. This is a user friendly, portable measuring device which is especially useful for quick determination of ferrite content. It contains a tiny tester with a probe and an etalon series. Because of its physical limitations its excitation level is very low. Therefore, the ferritscope is not able to measure the saturation polarization. It can derive the ferrite content only from the initial permeability of the sample.

\section{Results}

The AC magnetometer measurement was suitable to determine the normal magnetization curves of the specimens up to about $12,800 \mathrm{~A} / \mathrm{m}$ excitation field. Figure 1 shows some selected normal magnetization curves of the $0 \%, 31.3 \%$, and $61.9 \%$ deformed samples.

It can be seen that the normal magnetization curves cannot reach the saturation level because of the limited exciting magnetic field strength. Therefore, it was necessary to extrapolate the saturation polarization values. The base of the applied extrapolation method was the socalled multiphase hyperbolic model. The details of this model, the fitting and the extrapolation procedure were described in our previous papers [9-11].

Magnetic hysteresis loops of the samples were recorded by DC magnetometer measurement. The measured hysteresis loop of the initial sample (undeformed, without heat treatment) can be seen in Fig. 2. The highest excitation field of this robust DC magnetic yoke was about $270,000 \mathrm{~A} / \mathrm{m}$ which was enough to technically saturate the tested samples. The ferrite content was calculated from the maximal value of polarization which was considered as the saturation value.
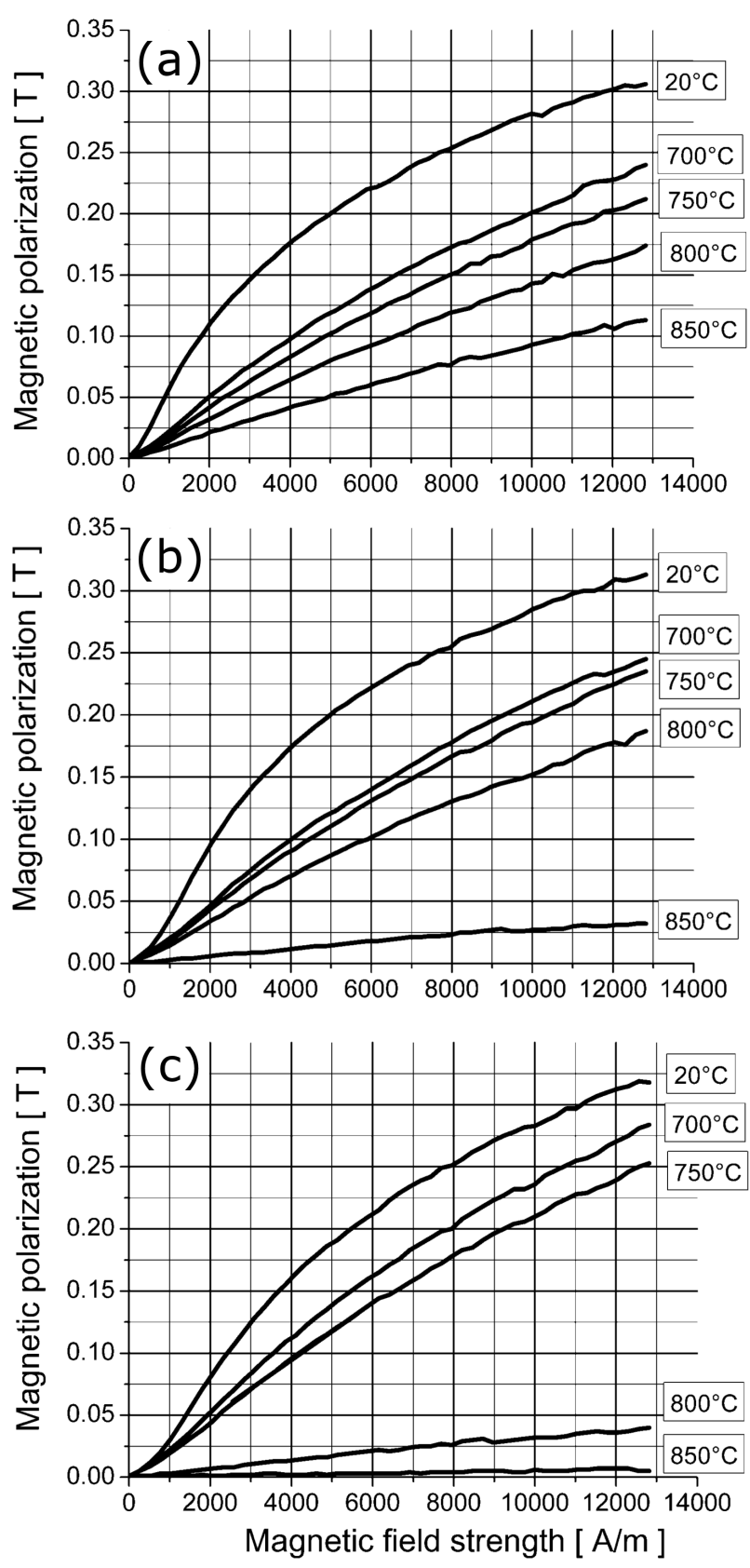

Fig. 1. Normal magnetization curves: (a) $\varepsilon=0 \%$, (b) $\varepsilon=31.3 \%$, (c) $\varepsilon=61.9 \%$ ).

The third applied measurement was the ferritscope which is the most user-friendly among the applied measuring devices. It directly indicates the ferrite content if the probe is put on the surface of the sample. Calibration before the measurement is necessary with an etalon series supplied by the producer. Because of its portability, user-friendliness, and easy use it is widespread in industry.

Figure 3 compares the values of the determined ferrite contents by the $\mathrm{AC}$ magnetometer after the extrapolation, the DC magnetometer and the ferritscope. 


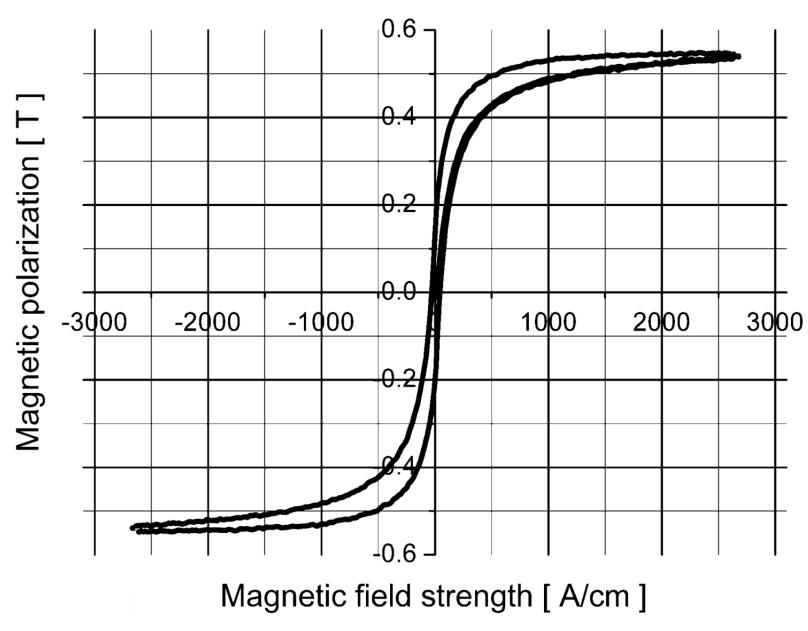

Fig. 2. Magnetic hysteresis loop of the sample in its initial state.

The plotted results belong to three samples of $0 \%, 31.3 \%$, and $61.9 \%$ rolling reduction. It can be seen that the results obtained by AC magnetometer are in good agreement with the results of the DC magnetometer measurement in case of each deformation rates. It can be seen that the stronger the cold rolling reduction was the lower the ferrite content was. However, it must be stressed that the ferritscope indicated significantly lower ferrite contents than the other two methods in case of deformed samples.

Figure 4 shows the ferrite content of the non-heat treated sample series as a function of the rolling reduction in case of the DC magnetometer and the ferritscope. The ferrite content is nearly constant for DC magnetometer measurement. In contrast, the ferrite values detected by the ferritscope decreases strongly as a function of the rolling reduction. It is known that the austenite is a stable phase in the 2507 type SDSS so the cold rolling has no effect on the ferrite content. Therefore, a measuring error of the ferritscope was observed in case of plastic deformed samples. If the extent of the cold rolling is strong this error can be about $25-30 \%$.

This inaccuracy of the Feritscope device is strongly associated with its physical principle. The Feritscope derives the ferrite content from the initial permeability of the sample. The initial permeability is influenced by the saturation polarization. Therefore, it can be used to determine the ferrite content of alloys. Unfortunately, the saturation polarization is influenced by the coercivity of the tested sample as well as it is explained in Fig. 5.

It is well known that the plastic deformation increases the coercive field because the induced dislocations make the domain wall movement more difficult. If the domain walls are prohibited in their motion the value of the coercive field increases.

Consequently, the normal magnetization curve of the deformed sample runs under the curve which belongs
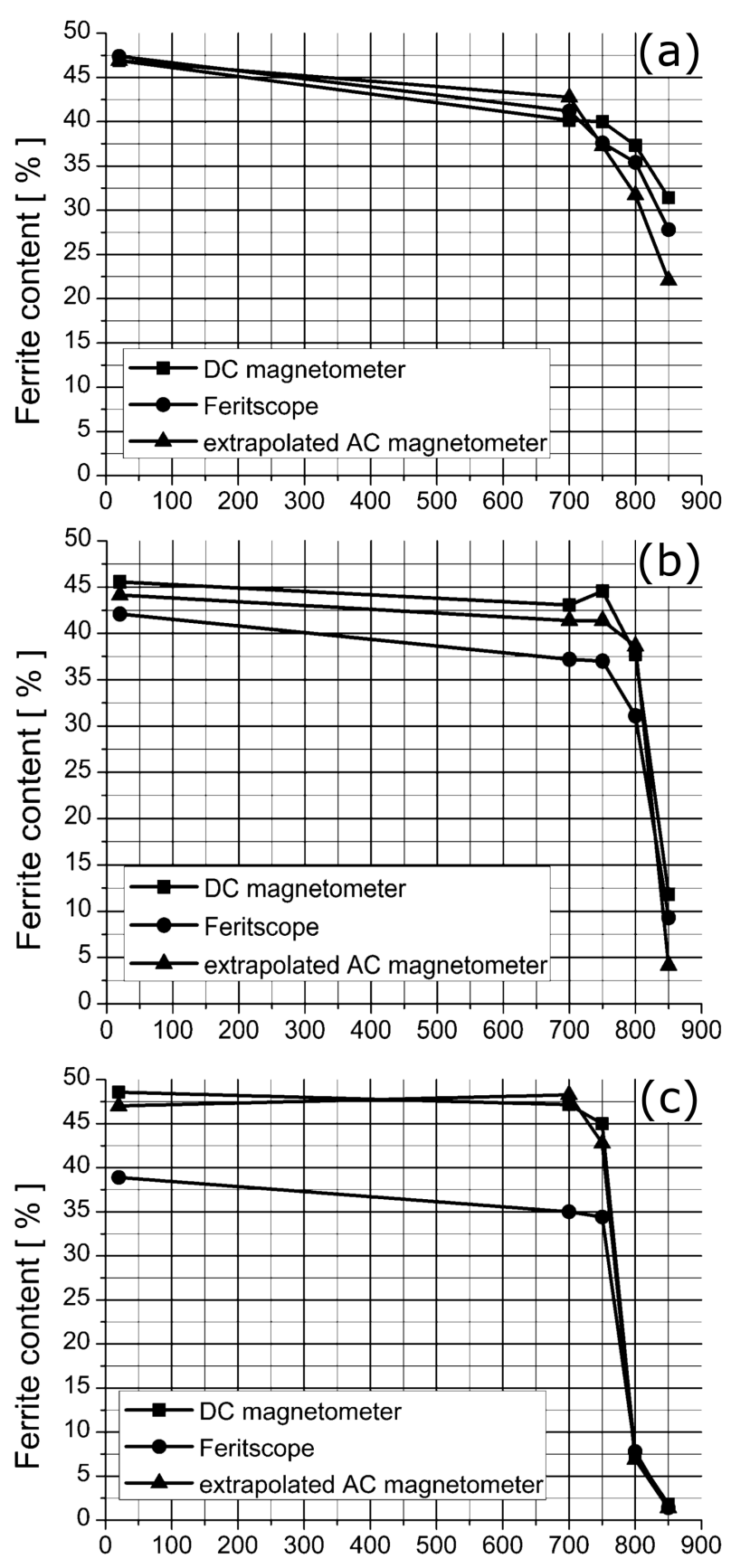

Heat treatment temperature $\left[{ }^{\circ} \mathrm{C}\right]$

Fig. 3. Ferrite content comparison of AC magnetometer, DC magnetometer and Feritscope: (a) $\varepsilon=0 \%$, (b) $\varepsilon=31.3 \%,(\mathrm{c}) \varepsilon=61.9 \%$ ).

to the undeformed specimen. Nevertheless, as it can be seen in Fig. 5 that the value of the initial permeability was strongly influenced by plastic deformation extent. Namely, it will be lower at the deformed sample compared to the undeformed specimen. Consequently, if the ferrite content of a deformed sample is derived from initial permeability it will be lower than its real value. 


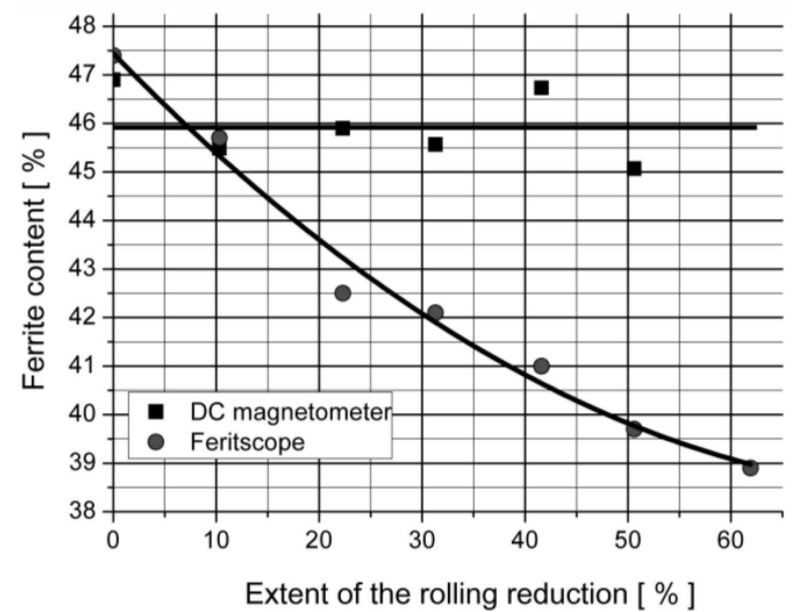

Fig. 4. Ferrite content of the non-heat treated sample series in function of the rolling reduction.

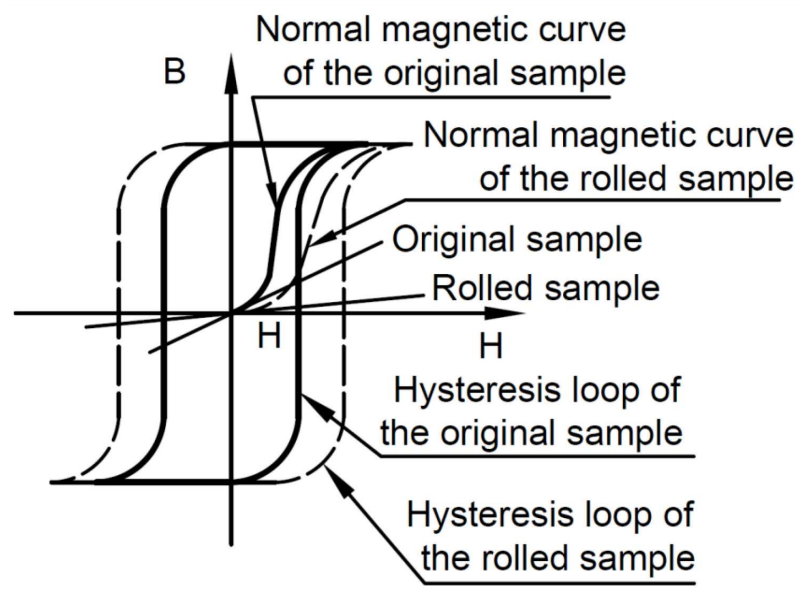

Fig. 5. The effect of the previous cold rolling for the ferrite measurement in case of the Feritscope.

\section{Summary}

Three electromagnetic methods, namely, AC magnetometer, DC magnetometer, and ferritscope were compared with each other. Methods were suitable to determine $\delta$-ferrite content of plastic deformed and heat treated SDSS samples.

It is believed that the most precise and reliable result was given by the DC magnetometer because of its physical principle. It can magnetize and measure the total volume of sample and it can excite the specimen into magnetic saturation.
$\mathrm{AC}$ magnetometer is not able to magnetize the samples into saturation. From the measured maximum polarization value, the saturation polarization can be extrapolated only. In this work the so-called multiphase hyperbolic model was used for this extrapolation. The obtained saturation polarization values are in good agreement with the measured results of the DC magnetometer.

Surprisingly, the feritscope detected significantly lower ferrite contents than the other two methods in case of plastic deformed samples. This difference was explained by the increases of coercivity and the change of the magnetization curves due to plastic deformation.

All the eddy-current based measuring devices like the applied ferritscope derive the ferrite content from the initial permeability which is influenced by the change of coercivity. Consequently, it can strongly affect the accuracy of the ferrite content measurement. Therefore, the application of ferritscope device requires special attention in case of sample series where the coercivity changes due to plastic deformation of other metallurgical effects.

\section{Acknowledgments}

The research reported in this paper was supported by the Higher Education Excellence Program of the Ministry of Human Capacities in the frame of Nanotechnology and Materials Science research area of Budapest University of Technology and Economics (BME FIKP-NAT).

\section{References}

[1] J. Charles, Steel Res. Int. 79, 455 (2008).

[2] M. Breda, K. Brunelli, F. Grazzi, A. Scherillo, I. Calliari, Metall. Mater. Trans. A 46, 577 (2015).

[3] F. Fiorillo, Measurement and Characterization of Magnetic Materials, Elsevier, Amsterdam 2004.

[4] G. Vértesy, I. Mészáros, I. Tomas, Nondestruct. Test. Eva. Int. 54, 107 (2013).

[5] I. Mészáros, J. Electr. Eng. 59, 86 (2008).

[6] F. Stablein, R. Steinitz, R. Arch Eisenhüttenwesen 8, 549 (1935).

[7] I. Mészáros, Mater. Sci. Forum 729, 109 (2013).

[8] I. Mészáros, J. Electr. Eng. 61, 1 (2010).

[9] Fischer, Feritscope FMP30 Measurement of the Ferrite Content in Austenitic and Duplex Steel.

[10] J. Takács, I. Mészáros, Physica B 403, 3137 (2008).

[11] I. Mészáros, Mater. Sci. Forum 721, 96 (2012). 